UDC 517.9

\title{
LOW-FREQUENCY PROPAGATION OF PLANE SCALAR WAVES THROUGH A PERIODIC ARRAY OF ARBITRARY VOLUMETRIC OBSTACLES
}

\section{ПОШИРЕННЯ НИЗЬКОЧАСТОТНИХ СКАЛЯРНИХ ХВИЛЬ НА ПЛОЩИНІ ЧЕРЕЗ ПЕРІОДИЧНИЙ МАСИВ ДОВІЛЬНИХ ПЕРЕІІКОД}

\author{
E. Scarpetta
}

Univ. Salerno

84084 Fisciano (SA), Italy

\section{A. Sumbatyan}

Research Inst. Mech. and Appl. Math.

Stachki Prospect 200/1, Rostov-on-Don 344090, Russia

In the context of wave propagation through gratings of periodically distributed obstacles, we use analytical approaches to derive explicit formulas for the scattering parameters in the low-frequency approximation. The geometric shape of the obstacles is arbitrary. Numerical solution of the main integral equations for assigned shapes will provide some graphs, and comparison with previous results will show that some of these are incorrect or hold under very restrictive conditions.

При розгляді поширення хвиль через гратку періодично розповсюджених перешкод за допомогою аналітичного підходу отримано явні формули для параметрів розсіяння при низькочастотній апроксимації. Геометрична форма перешкод є довільною. На основі чисельного розв'язку основних інтегральних рівнянь для вибраних форм побудовано графіки, а порівняння з попередніми результатами показує, щз деякі з них не підтверджуються, а інші мають місце за досить суворих обмежень.

1. Introduction. The importance of scattering problems through gratings of obstacles variously distributed inside a medium is well known in many practical applications regarding mechanical, acoustic or electromagnetic sciences. The books of Krautkramers [1] and Jones [2] can be usefully referred to for a survey of the researches devoted to such topics. These investigations can be performed following either purely numerical or analytical points of view, the latter ones being more involved when regular (periodic) distributions of obstacles are concerned; see [3] where a number of references for both these approaches can be found. In every case, it is assumed very often that the frequency of the incident wave (perhaps together with the characteristic size of the obstacles) is small, giving typically rise to a weak interaction regime in which approximate results can be established.

Of course, analytical methods are valuable since they provide explicit formulas for the relevant scattering parameters, from which a correct limit for low frequencies can be easily extracted. Actually, up to today, this has been made only for thin (slit-type) obstacles; see [2, $4-8$ ] for an outline of such analytical procedures.

Some authors also claimed explicit results for low frequencies in the case of rectangular obstacles periodically distributed $[9,10]$. Of course, this case (geometrically equivalent to a

(C) E. Scarpetta, M. A. Sumbatyan, 2003

ISSN 1562-3076. Нелінійні коливання, 2003, m. 6, N으 
screen of finite thickness with regular gaps) is more simple with respect to that of arbitrarily shaped obstacles, because it allows an (analytical) approach in terms of Fourier series [3, 11]. Nevertheless, we will show below that such results (echoed in [11]) are incorrect.

For an arbitrary (volumetric) shape, some interesting analytical results were obtained by Lamb [4], Twersky [12] and Miles [13]. These authors operate starting from an a priori known solution of the problem regarding an ideal incompressible fluid flow around a single obstacle of the given shape, solution which is clearly independent of frequency. Unfortunately, this method works well under the assumption of weak reflection between the wave and the obstacle, thus requiring not only a low frequency, but also the smallness of the obstacle (actually, we will show below in concrete examples that, even in the low-frequency range, the Lamb-Twersky-Miles approximation holds good enough for only very small obstacles).

Besides what already has been mentioned about previous results, the aim of the present paper is to construct analytically a correct low-frequency limit from explicit formulas in scattering (scalar) problems involving periodic gratings of arbitrary volumetric obstacles. Particular attention will be devoted to re-considering the case of rectangular scatterers, which admits an alternative procedure and thus permits an interesting comparison between the two methods of solution (along with reciprocal testing of efficiency).

Even if the framework can be directly applied to similar problems in acoustics or electromagnetism, we prefer to address the problem in elastic context, in which the scalar wave field and obstacles concerned are interpreted as anti-plane displacement and special defects in the (solid) structure, respectively.

2. Mathematical formulation. We consider the anti-plane normal penetration of a harmonic plane wave of SH-type into an unbounded two-dimensional elastic medium in which an infinite periodic array of equal defects with arbitrary shape is present (see Fig. 1). Denoted by $d$ the period of this vertical grating, the natural symmetry of the problem entitles us to restrict the attention to a single layer $0<y_{2}<d$ containing an obstacle $D$; let $l$ be the regular line surrounding $D$ (to which can be applied the plane Green theorem). In the assumed harmonic regime, all field variables have the common factor $e^{-i \omega t}$, that will be omitted in the sequel.

Thus, the incident (scalar) wave of unitary amplitude has the form $e^{i k y_{1}}$ and gives rise to a scattered (stationary) field $\varphi_{s c}\left(y_{1}, y_{2}\right)$ satisfying the Helmholtz equation throughout the layer except $D$ :

$$
\Delta_{y} \varphi_{s c}+k^{2} \varphi_{s c}=0, \quad \Delta_{y}=\partial^{2} / \partial y_{1}^{2}+\partial^{2} / \varphi y_{2}^{2}
$$

Above, $k$ is the wave number and $\omega$ the circular frequency; of course, $\omega / k$ gives the (transverse) wave speed of the material in concern. Writing

$$
\varphi\left(y_{1}, y_{2}\right)=\varphi_{s c}\left(y_{1}, y_{2}\right)+e^{i k y_{1}}
$$

for the total wave field, which also satisfies the Helmholtz equation, the periodicity requires that

$$
\varphi\left(y_{1}, 0\right)=\varphi\left(y_{1}, d\right), \quad \frac{\partial \varphi}{\partial y_{2}}\left(y_{1}, 0\right)=\frac{\partial \varphi}{\partial y_{2}}\left(y_{1}, d\right)
$$




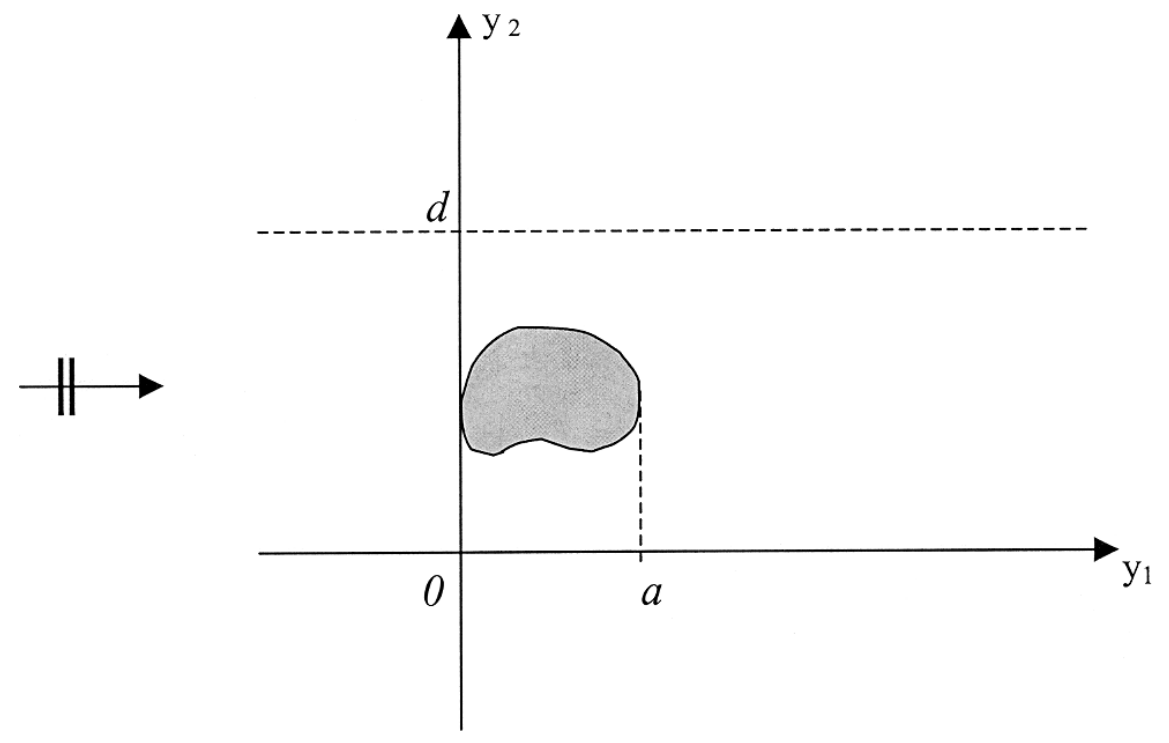

Fig. 1. Normal penetration of a plane scalar wave into a periodic array of arbitrary defects.

Moreover, in view of the low-frequency regime to be considered, we can assume that the total field in the far left and right parts of the layer be expressed in the following (one-mode) form:

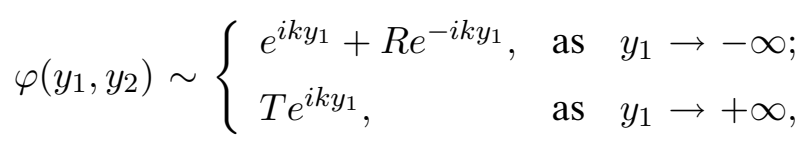

where $R$ and $T$ represent the reflection and transmission coefficients of the structure, respectively; cf. $[6-8,11,13]$. These are the scattering parameters for which (approximate) formulas are searched showing explicitly the dependence on the frequency.

Since the contour of the defects cannot sustain (tangential) stress, we put, as basic boundary condition of the problem, the vanishing of the normal derivative of the total field along $l$,

$$
\left.\frac{\partial \varphi}{\partial n}\right|_{l}=0
$$

Now, we need to consider an appropriate Green's function $\Phi=\Phi(y ; x)$ for the given layer (henceforth, we will find convenient to put simply $y$ or $x$ for the pair $\left(y_{1}, y_{2}\right)$ or $\left(x_{1}, x_{2}\right)$ ); it should satisfy the nonhomogeneous Helmholtz equation

$$
\Delta_{y} \Phi(y ; x)+k^{2} \Phi(y ; x)=-\delta\left(y_{1}-x_{1}\right) \delta\left(y_{2}-x_{2}\right)
$$

along with the periodicity conditions

$$
\Phi\left(y_{1}, 0 ; x\right)=\Phi\left(y_{1}, d ; x\right), \quad \frac{\partial \Phi}{\partial y_{2}}\left(y_{1}, 0 ; x\right)=\frac{\partial \Phi}{\partial y_{2}}\left(y_{1}, d ; x\right) .
$$


To this aim, we resort to a work by Van den Berg and Voorman [14], where in a similar context (but addressed to purely numerical results) such a function is constructed as

$$
\begin{gathered}
\Phi(y ; x)=\frac{i e^{i k\left|y_{1}-x_{1}\right|}}{2 k d}+\sum_{n=1}^{+\infty} \frac{e^{-\beta_{n}\left|y_{1}-x_{1}\right|} \cos \left[(2 \pi n / d)\left(y_{2}-x_{2}\right)\right]}{\beta_{n} d}, \\
\beta_{n}=\sqrt{(2 \pi n / d)^{2}-k^{2}} .
\end{gathered}
$$

Of course, the aimed low-frequency approximation entitles us to consider real and positive numbers $\beta_{n}$, so that the following asymptotic expression of $\Phi$ holds at large distances from the defects' array:

$$
\Phi(y ; x) \sim \frac{i e^{i k\left|y_{1}-x_{1}\right|}}{2 k d} \quad\left(\text { as } x_{1} \text { or } y_{1} \rightarrow \pm \infty\right)
$$

Considering the boundary condition (2.5), classical results of potential theory $[2,15]$ can be used to provide the scattered field in the layer (except D) with the following integral representation over the contour $l$ in terms of the normal derivative of the Green's function:

$$
\varphi_{s c}(x)=\varphi(x)-e^{i k x_{1}}=\int_{l}\left[\varphi(y) \frac{\partial \Phi}{\partial n_{y}}(y ; x)\right] d l_{y}, \quad x \text { is outside of } D
$$

( $n_{y}$ is the external unit normal to $D$ ). Aiming to let $x_{1} \rightarrow \pm \infty$ in Eq. (2.10), we can calculate $\frac{\partial \Phi}{\partial n_{y}}$ from Eq. (2.9) as follows:

$$
\frac{\partial \Phi}{\partial n_{y}} \approx \frac{\partial \Phi}{\partial y_{1}} n_{1}(y) \approx \frac{e^{i k\left|y_{1}-x_{1}\right|}}{2 d} n_{1}(y) \operatorname{sign}\left(x_{1}-y_{1}\right) \quad\left(\text { as } \quad x_{1} \rightarrow \pm \infty\right) .
$$

Therefore, by Eq. (2.10) when $x_{1} \rightarrow-\infty$ or $+\infty$ and Eq. $(2.4)_{1}$ or $(2.4)_{2}$, we get, respectively,

$$
\begin{gathered}
\varphi_{s c}(x) \approx-\int_{l} \varphi(y) \frac{e^{i k\left(y_{1}-x_{1}\right)}}{2 d} n_{1}(y) d l_{y} \approx R e^{-i k x_{1}}, \quad \text { as } x_{1} \rightarrow-\infty, \\
\varphi_{s c}(x) \approx \int_{l} \varphi(y) \frac{e^{i k\left(x_{1}-y_{1}\right)}}{2 d} n_{1}(y) d l_{y} \approx(T-1) e^{i k x_{1}}, \quad \text { as } x_{1} \rightarrow+\infty,
\end{gathered}
$$

from which the following expressions for the reflection and transmission coefficients are easily derived:

$$
R=-\frac{1}{2 d} \int_{l} \varphi(y) e^{i k y_{1}} n_{1}(y) d l_{y}, \quad T=1+\frac{1}{2 d} \int_{l} \varphi(y) e^{-i k y_{1}} n_{1}(y) d l_{y}
$$


Note that the dependence on $k$ is also involved (implicitly) in $\varphi(y)$, whose restriction to $l$ actually remains the only unknown of the problem. Thus, in order to find a (boundary) integral equation to be solved for $\varphi$, we let $x \rightarrow Y \in l$ in Eq. (2.10) and invoke well-known properties of the "double layer" integral in the potential theory, to obtain

$$
\frac{1}{2} \varphi(Y)-\int_{l}\left[\varphi(y) \frac{\partial \Phi}{\partial n_{y}}(y ; Y)\right] d l_{y}=e^{i k Y_{1}}, \quad Y=\left(Y_{1}, Y_{2}\right) \in l
$$

which gives the searched equation (cf. [14]).

3. Explicit results for the scattering coefficients in the low-frequency limit. We now aim to derive the (analytical) limit of Eqs. (2.13), (2.14) as $\omega \sim k \rightarrow 0$. Our strategy is that to neglect terms of order $O\left(k^{2}\right)$ wherever they appear. We firstly need to evaluate the limiting form of the Green's function from Eq. (2.8); putting there $\beta_{n} \approx 2 \pi n / d$, we get

$$
\begin{aligned}
\Phi(y ; x) \simeq & \frac{i e^{i k\left|y_{1}-x_{1}\right|}}{2 k d}+\frac{1}{2 \pi} \sum_{n=1}^{\infty} \frac{e^{-(2 \pi n / d)\left|y_{1}-x_{1}\right|} \cos \left[(2 \pi n / d)\left(y_{2}-x_{2}\right)\right]}{n}= \\
= & \frac{i e^{i k\left|y_{1}-x_{1}\right|}}{2 k d}+\frac{\left|y_{1}-x_{1}\right|}{2 d}- \\
& -\frac{1}{4 \pi} \ln \left\{2\left[\cosh \frac{2 \pi\left(y_{1}-x_{1}\right)}{d}-\cos \frac{2 \pi\left(y_{2}-x_{2}\right)}{d}\right]\right\}, \quad \text { as } k \rightarrow 0,
\end{aligned}
$$

where the summation formula

$$
\sum_{n=1}^{\infty} \frac{e^{-n \xi} \cos (n \zeta)}{n}=\frac{\xi}{2}-\frac{1}{2} \ln [2(\cosh \xi-\cos \zeta)]
$$

has been used. As a consequence, the limit of $\frac{\partial \Phi}{\partial n_{y}}=\frac{\partial \Phi}{\partial y_{1}} n_{1}(y)+\frac{\partial \Phi}{\partial y_{2}} n_{2}(y)$ in Eq. (2.14) can be obtained as follows:

$$
\begin{aligned}
\frac{\partial \Phi}{\partial n_{y}}(y ; Y) \simeq & \frac{1}{2 d}\left(1-e^{i k\left|y_{1}-Y_{1}\right|}\right) \operatorname{sign}\left(y_{1}-Y_{1}\right) n_{1}(y)- \\
& -\frac{1}{2 d} \frac{\sinh \left[(2 \pi / d)\left(y_{1}-Y_{1}\right)\right] n_{1}(y)+\sin \left[(2 \pi / d)\left(y_{2}-Y_{2}\right)\right] n_{2}(y)}{\cosh \left[(2 \pi / d)\left(y_{1}-Y_{1}\right)\right]-\cos \left[(2 \pi / d)\left(y_{2}-Y_{2}\right)\right]}, \quad y, Y \in l,
\end{aligned}
$$

from which, with error $O\left(k^{2}\right)$, we finally get

$$
\frac{\partial \Phi}{\partial n_{y}}(y ; Y)=-\frac{i k}{2 d}\left(y_{1}-Y_{1}\right) n_{1}(y)-K_{0}(y, Y), \quad \text { as } k \rightarrow 0,
$$


where the kernel

$$
K_{0}(y, Y)=\frac{1}{2 d} \frac{\sinh \left[(2 \pi / d)\left(y_{1}-Y_{1}\right)\right] n_{1}(y)+\sin \left[(2 \pi / d)\left(y_{2}-Y_{2}\right)\right] n_{2}(y)}{\cosh \left[(2 \pi / d)\left(y_{1}-Y_{1}\right)\right]-\cos \left[(2 \pi / d)\left(y_{2}-Y_{2}\right)\right]}, \quad y, Y \in l,
$$

is real and does not contain $k$.

Thus, the main integral equation (2.14) at $k \rightarrow 0$ can be written (with error $O\left(k^{2}\right)$ ) as:

$$
\frac{1}{2} \varphi(Y)+\int_{l} K_{0}(y, Y) \varphi(y) d l_{y}=1+i k Y_{1}-\frac{i k}{2 d}\left(g_{1}-g_{0} Y_{1}\right), \quad Y \in l
$$

where

$$
g_{0} \equiv \int_{l} \varphi(y) n_{1}(y) d l_{y}, \quad g_{1} \equiv \int_{l} y_{1} \varphi(y) n_{1}(y) d l_{y} .
$$

Now, writing formally the left-hand term above as $\left(\frac{1}{2} I+K_{0}\right) \varphi(Y)$, it is clear by linearity that if one can solve the two integral equations

$$
\left(\frac{1}{2} I+K_{0}\right) h_{0}(Y)=1, \quad\left(\frac{1}{2} I+K_{0}\right) h_{1}(Y)=Y_{1}, \quad Y \in l
$$

both of them real and not containing $k$, then a solution of Eq. (3.5) can be constructed as

$$
\varphi(y)=\left(1-\frac{i k}{2 d} g_{1}\right) h_{0}(y)+\frac{i k}{2 d}\left(2 d+g_{0}\right) h_{1}(y), \quad y \in l .
$$

To find constants $g_{0}$ and $g_{1}$, let us integrate twice Eq. (3.7) with respect to $l$ after multiplying by $n_{1}(y)$ and $y_{1} n_{1}(y)$. We get, respectively,

$$
\begin{aligned}
& g_{0}=\left(1-\frac{i k}{2 d} g_{1}\right) H_{00}+\frac{i k}{2 d}\left(g_{0}+2 d\right) H_{10}, \\
& g_{1}=\left(1-\frac{i k}{2 d} g_{1}\right) H_{01}+\frac{i k}{2 d}\left(g_{0}+2 d\right) H_{11},
\end{aligned}
$$

where

$$
\begin{aligned}
H_{00}=\int_{l} h_{0}(y) n_{1}(y) d l_{y}, & H_{10}=\int_{l} h_{1}(y) n_{1}(y) d l_{y}, \\
H_{01}=\int_{l} y_{1} h_{0}(y) n_{1}(y) d l_{y}, & H_{11}=\int_{l} y_{1} h_{1}(y) n_{1}(y) d l_{y}
\end{aligned}
$$


are real constants not containing $k$, that can be calculated after solving the auxiliary integral equations (3.6). Solution of the linear system (3.8) gives, letting $k \rightarrow 0$ and neglecting terms $O\left(k^{2}\right)$ in numerator and denominator,

$$
\begin{gathered}
g_{0}=\frac{H_{00}+i k H_{10}}{1+(i k / 2 d)\left(H_{01}-H_{10}\right)}, \\
g_{1}=\frac{H_{01}+i k\left[H_{11}+(1 / 2 d)\left(H_{00} H_{11}-H_{10} H_{01}\right)\right]}{1+(i k / 2 d)\left(H_{01}-H_{10}\right)} .
\end{gathered}
$$

On the other side, letting $k \rightarrow 0$ and using Eqs. (3.5b), Eqs. (2.13) become (with error $\left.O\left(k^{2}\right)\right)$

$$
R=-\frac{1}{2 d}\left(g_{0}+i k g_{1}\right), \quad T=1+\frac{1}{2 d}\left(g_{0}-i k g_{1}\right)
$$

By substituting $g_{0}$ and $g_{1}$ from Eqs. (3.10), we easily get

$$
R=-\frac{H_{00}+i k\left(H_{10}+H_{01}\right)}{2 d+i k\left(H_{01}-H_{10}\right)}, \quad T=\frac{H_{00}+2 d}{2 d+i k\left(H_{01}-H_{10}\right)}
$$

(neglecting terms $O\left(k^{2}\right)$ in the numerator).

Of course, from a physical standpoint, the reflection coefficient should vanish when $k$ does, and this implies that the constant $H_{00}$ must be null identically ${ }^{1}$. Thus, the above explicit formulas finally become in the present low-frequency approximation :

$$
\begin{gathered}
R=-\frac{i k\left(H_{10}+H_{01}\right)}{2 d+i k\left(H_{01}-H_{10}\right)}, \\
T=\frac{2 d}{2 d+i k\left(H_{01}-H_{10}\right)} .
\end{gathered}
$$

We remark that Eqs. (3.13) hold without any assumption of smallness of the defects; cf. [13].

4. Case of rectangular obstacles. The above results hold for an arbitrary geometric shape of the defects (this shape being involved only in Eqs. (3.6) and (3.9)), and of course can be as well applied to rectangular defects. Such a particular case has a special importance in scattering problems since it formally amounts to consider a screen of finite thickness inside the medium having regular gaps periodically distributed. Apart from approaches of numerical or engineering type in acoustic context $[9,10]$, this case has been treated analytically in [11] by means of suitable Fourier expansions of the wave field in the three main regions of the layer (vertically centered around the opening between adjacent obstacles, rather than around the obstacle itself; see Fig. 2). By imposing the continuity of the wave field and of the stress' $z x$-component through neighbouring regions, we arrived at the following formulas for the reflection and transmission

\footnotetext{
${ }^{1}$ This will be confirmed by numerical calculations (Section 5).
} 


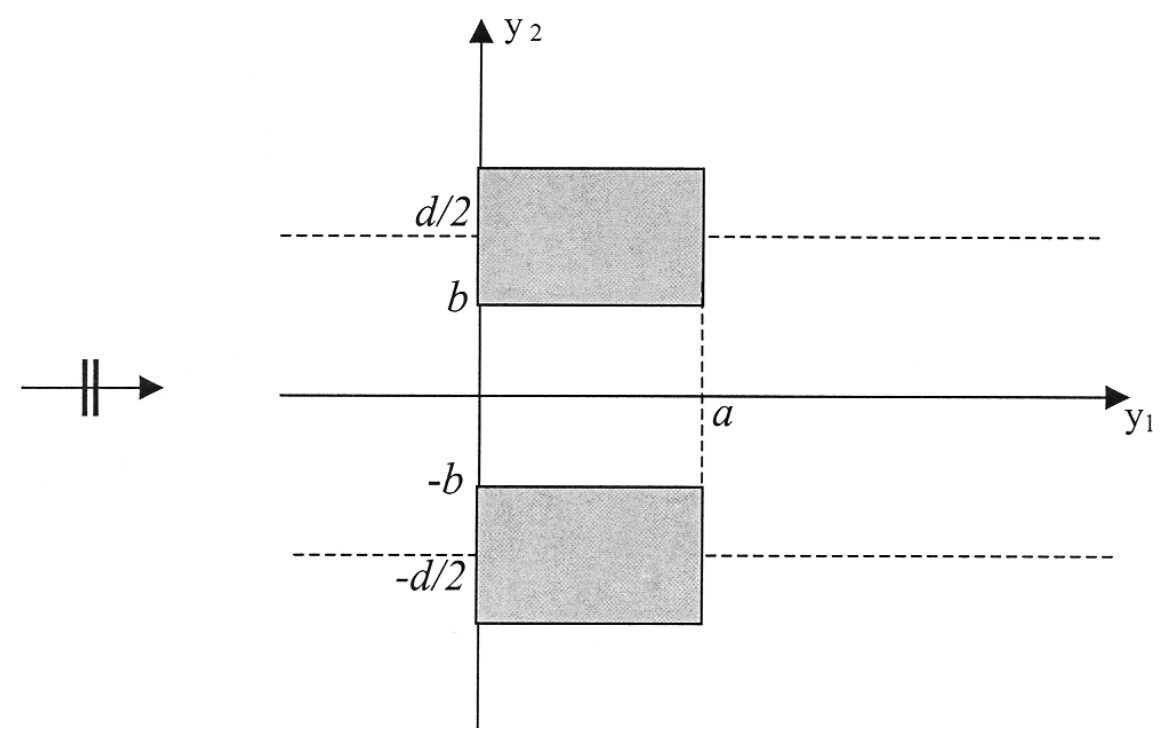

Fig. 2. Normal penetration of a plane scalar wave into a periodic array of rectangular defects $a \times(d-2 b)$.

coefficients (defined as in Eqs. (2.4) in the context of the same far-field propagation of onemode type):

$$
\begin{gathered}
R=1-\frac{G^{+}+G^{-}}{2 i k d}, \quad t=\frac{G^{+}-G^{-}}{2 i k d}, \\
G^{ \pm}=\frac{-2 S^{ \pm}}{1-\left[\frac{\cos (k a) \mp 1}{2 b k \sin (k a)}+\frac{1}{i k d}\right] S^{ \pm}}, \quad S^{ \pm}=\int_{-b}^{b} s^{ \pm}\left(y_{2}\right) d y_{2},
\end{gathered}
$$

where $(-b, b)$ is the opening between adjacent rectangles, of which $a$ is the length, and $s^{ \pm}\left(y_{2}\right)$ are two (real) functions solving the integral equations

$$
\begin{aligned}
\int_{-b}^{b} s^{ \pm}(t) & \left\{\sum_{n=1}^{\infty} \frac{1}{\sqrt{(\pi n)^{2}-(k d / 2)^{2}}} \cos \left[\frac{2 \pi n}{d}\left(y_{2}-t\right)\right]+\right. \\
& \left.+\frac{\operatorname{ch~} \sqrt{(\pi n a / b)^{2}-(k a)^{2}} \mp 1}{\sqrt{(\pi n)^{2}-(k b)^{2}} \operatorname{sh} \sqrt{(\pi n a / b)^{2}-(k a)^{2}}} \cos \left[\frac{\pi n}{b}\left(y_{2}-t\right)\right]\right\} d t=1, \quad\left|y_{2}\right|<b
\end{aligned}
$$

Now, we aim to derive the low-frequency limit for these formulas. Constants $S^{ \pm}$in Eqs. (4.1) depend on $k$ via the kernels of Eqs. (4.2), so that to extract correct behaviour of $G^{ \pm}, R$, $T$, when $k \rightarrow 0$, we need first to study this limit for Eqs. (4.2). Neglecting there terms $O\left(k^{2}\right)$ under 
square roots, and using the summation formula

$$
\sum_{n=1}^{\infty} \cos \frac{2 \pi n \xi / d}{n}=-\ln \left|2 \sin \frac{\pi \xi}{d}\right|
$$

we easily get the integral equations, free of frequency parameter, to be satisfied by the functions $s^{ \pm}$in the low-frequency approximation,

$$
\frac{1}{\pi} \int_{-b}^{b} s^{ \pm}(t)\left\{\sum_{n=1}^{\infty} \frac{\operatorname{ch}(\pi n a / b) \mp 1}{n \operatorname{sh}(\pi n a / b)} \cos \left[\frac{\pi n}{b}\left(y_{2}-t\right)\right]-\ln \left|2 \sin \frac{\pi\left(y_{2}-t\right)}{d}\right|\right\} d t=1, \quad\left|y_{2}\right|<b .
$$

Let us go back to Eqs. (4.1b) for constants $G^{ \pm}$, in which the (real) values for $S^{ \pm}$are now taken from the solutions of Eqs. (4.4), so that the dependence on $k$ actually appears only in the square bracket. To get the limit as $k \rightarrow 0$, we must consider $G^{+}$and $G^{-}$separately; in $G^{-}$we have

$$
1-\left[\frac{\cos (k a)+1}{2 b k \sin (k a)}+\frac{1}{i k d}\right] S^{-}=1-\frac{\cot (k a / 2)}{2 b k} S^{-}-\frac{1}{i k d} S^{-} \simeq-\left(\frac{\cot (k a / 2)}{2 b k}+\frac{1}{i k d}\right) S^{-},
$$

which gives

$$
G^{-} \simeq \frac{2 S^{-}}{\frac{i(d / 2) \cot (k a / 2)+b}{i b k d} S^{-}}=\frac{2 b k d}{(d / 2) \cot (k a / 2)-i b}, \quad \text { as } \quad k \rightarrow 0 .
$$

On the contrary, in $G^{+}$we have

$$
1-\left[\frac{\cos (k a)-1}{2 b k \sin (k a)}+\frac{1}{i k d}\right] S^{+}=1+\frac{\tan (k a / 2)}{2 b k} S^{+}-\frac{1}{i k d} S^{+}
$$

and therefore such a constant remains unchanged as

$$
G^{+}=-\frac{2 b k d}{(d / 2) \tan (k a / 2)+i b+b k d / S^{+}}
$$

also in the limit $k \rightarrow 0$.

Using these values for $G^{+}, G^{-}$, formulas (4.1a) have the following limits as $k \rightarrow 0$ (with the same approximation as in Sect. 3):

$$
\begin{gathered}
R=-\frac{i k\left[\left(d^{2} / 4\right)-b^{2}+\left(b d^{2} / a S^{+}\right)\right]}{b d / a-i k\left[\left(d^{2} / 4\right)+b^{2}+\left(b d^{2} / a S^{+}\right)\right]}, \\
T=\frac{b d / a}{b d / a-i k\left[\left(d^{2} / 4\right)+b^{2}+\left(b d^{2} / a S^{+}\right)\right]}
\end{gathered}
$$

(cf. $[10,11]$, Sect. 4).

ISSN 1562-3076. Нелінійні коливання, 2003, m. 6, № 4 


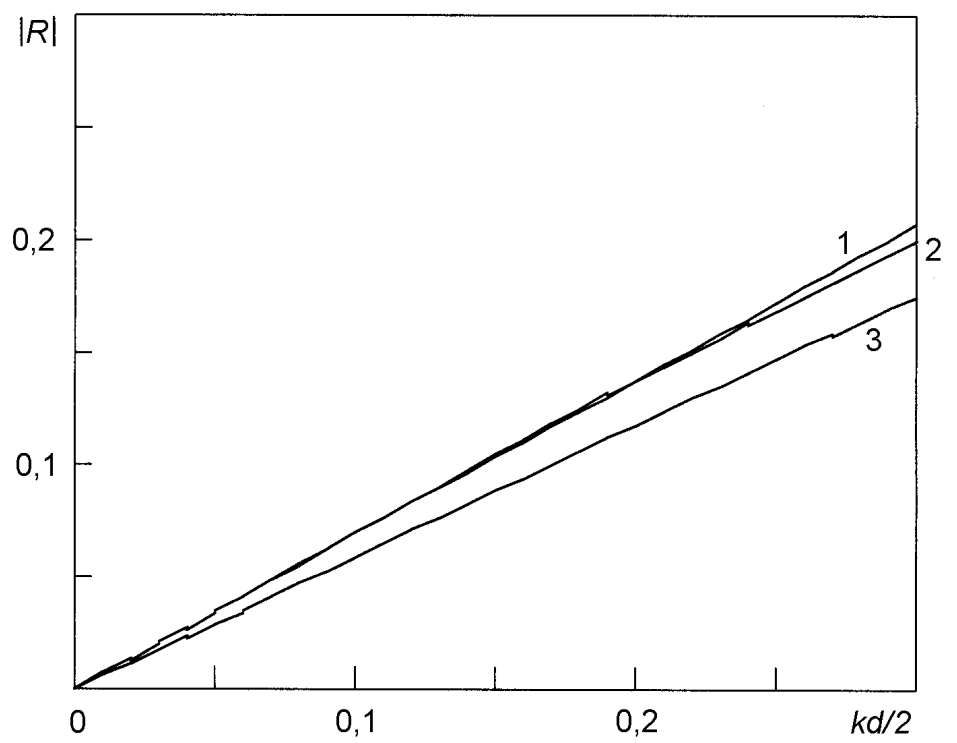

Fig. 3. Reflection coefficient $|R|$ vs. frequency parameter $k d / 2$ for circular defects of diameter $a=0,5 d$ in the case of small frequencies: $1-$ analytical solution (3.13a), 2 - exact solution, 3 - Miles' solution.

5. Numerical investigation and comparison with previous results. First of all, we should remark that both the pairs of formulas (3.13) and (4.6) identically satisfy the well-known balance of energies [15]

$$
|R|^{2}+|T|^{2}=1
$$

with error $O\left(k^{2}\right)$. So, for what follows, it is sufficient to consider one only of the scattering coefficients, for example, the reflection's one.

By applying a direct numerical method to the integral equations (3.6) for some particular shapes and disposition of the defects, we obtained values for all constants $H$ defined in Eqs. (3.9). As the physics requires, $H_{00}$ turns out to be always vanishing. Behavior of the function $|R|$ versus (small values of) the frequency parameter, from Eq. (3.13a), is reflected as line 1 in all Figures 3-6. Validity of Eqs. (2.4) and (2.9) is guaranteed until $k d / 2<\pi$; cf. [13]. We can observe a good agreement (better for smaller obstacles) with line 2 reflecting the exact solution, as derived numerically from Eqs. (2.14), (2.13) $)_{1}$; in any case, the initial slope is the same. Miles' solution [13] gives a (perfectly) linear behavior, reflected as line 3 in Figures 3-6, which appears far from lines 1, 2 (indeed, farther for larger obstacles) and with different initial slope. We note that Miles in [13] also constructed more approximate (not linear) formulas, but he used a rather artificial procedure from both the mathematical and physical standpoints (see Sect. 3 in that paper).

Figures 7, 8 are concerned with defects of rectangular shape. In them, we compare the analytical approach developed in this paper with the alternative one based on Fourier expansion and continuity conditions [11], and expressed by Eqs. (4.6). The range of frequency has been 


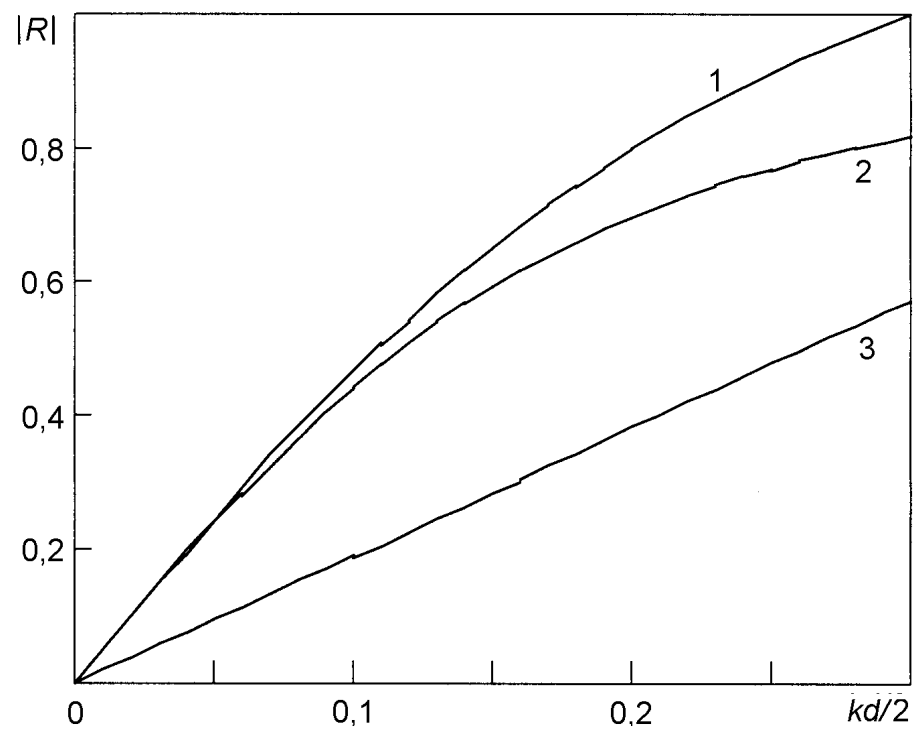

Fig. 4. Reflection coefficient $|R|$ vs. frequency parameter $k d / 2$ for circular defects of diameter $a=0,9 d$ in the case of small frequencies: $1-$ analytical solution (3.13a), 2 - exact solution, 3- Miles' solution.

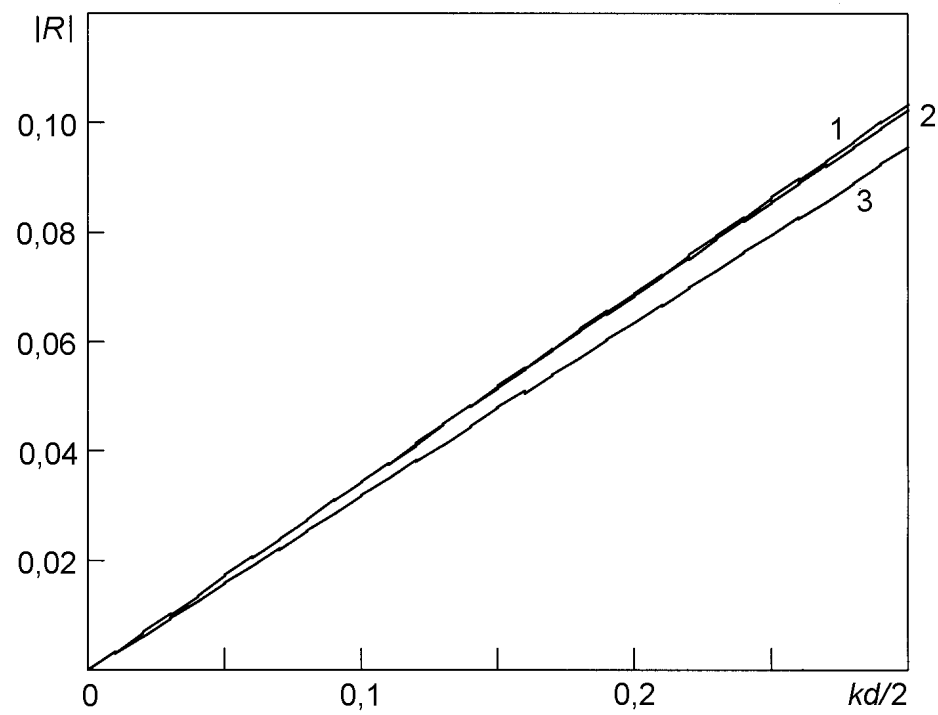

Fig. 5. Reflection coefficient $|R|$ vs. frequency parameter $k d / 2$ for elliptical defects of semi-axes $\alpha_{1} / \alpha_{2}=2\left(\alpha_{1}=0,5 d\right)$ rotated at $45^{\circ}$, in the case of small frequencies: 1 - analytical solution (3.13a), 2 - exact solution, 3 - Miles' solution. 


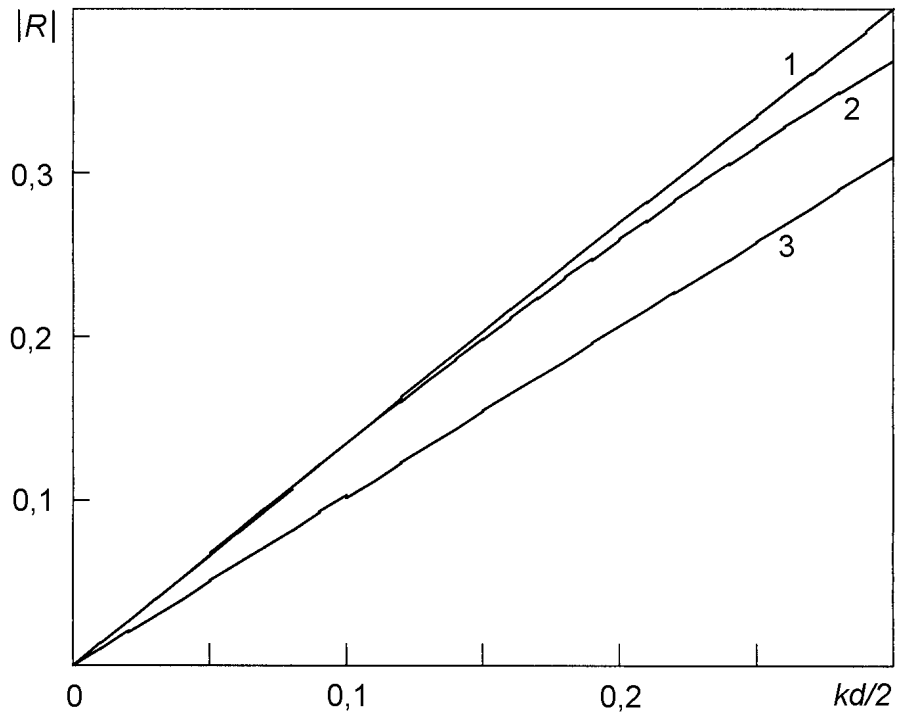

Fig. 6. Reflection coefficient $|R|$ vs. frequency parameter $k d / 2$ for elliptical defects of semi-axes $\alpha_{1} / \alpha_{2}=2\left(\alpha_{1}=0,9 d\right)$ rotated at $45^{\circ}$, in the case of small frequencies: 1 - analytical solution (3.13a), 2 - exact solution, 3 - Miles' solution.

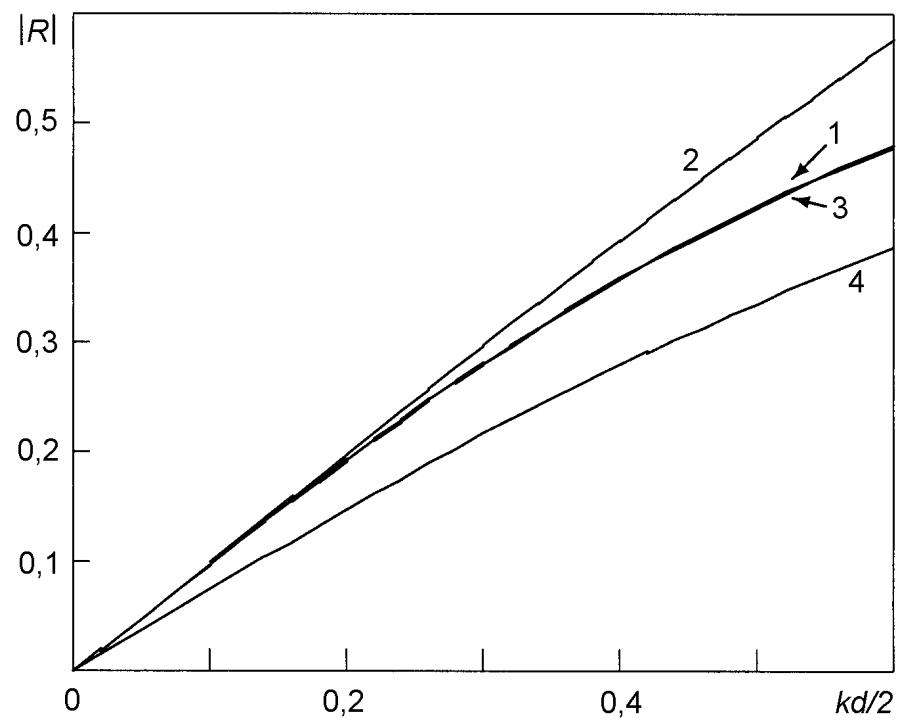

Fig. 7. Reflection coefficient $|R|$ vs. frequency parameter $k d / 2$ for quadratic defects with $a=0,5 d$ in the case of small frequencies: $1-$ exact solution, 2 - analytical solution (3.13a), 3 - analytical solution (4.6a), $4-$ engineering solution [10]. 


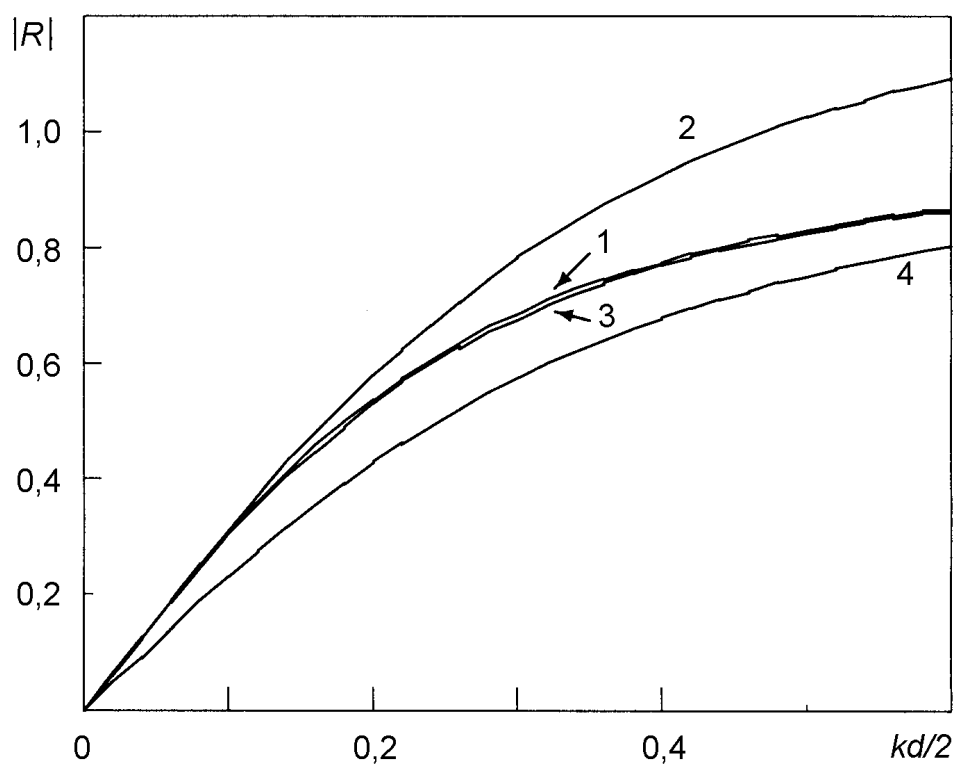

Fig. 8. Reflection coefficient $|R|$ vs. frequency parameter $k d / 2$ for rectangular defects with $a=0,5 d, b=0,1 d$ in the case of small frequencies: 1 - exact solution, 2 - analytical solution (3.13a), 3 - analytical solution (4.6a), 4 - engineering solution [10].

enlarged to improve comparison. Equations (4.2) hold until $k d / 2<\pi$. Line 1 reflects the exact solution, numerically obtained from Eqs. (2.14), (2.13) $)_{1}$ and practically coincident, in the given range, with the exact and one-mode solutions developed in [11] (see lines 1, 2 in Fig. 2 of that paper). Lines 2 and 3 reflect the low-frequency solutions analytically given by Eq. (3.13a) and (4.6a), respectively; the values for $S^{+}$are derived by numerical solution of Eq. (4.4). We can observe that the approach of [11], built up for only rectangular defects, is more precise throughout the considered range. Line 4, finally, reflects the explicit solution derived in [10] by means of an engineering approach confined to low-frequency; we could claim as incorrect this solution, since the initial slope of the line is quite different from that of other lines (cf. also line 3 in Fig. 2 of [11]).

1. Krautkramer J., Krautkramer H. Ultrasonic testing of materials. - 3rd ed. - New York: Springer, 1983.

2. Jones D. S. Acoustic and electromagnetic waves. - Oxford: Clarendon Press, 1986.

3. Scarpetta E., Sumbatyan M. A. In-plane wave propagation through elastic solids with a periodic array of rectangular defects // J. Appl. Mech. - 2002. - 69. - P. 179-188.

4. Lamb H. Hydrodynamics. - Cambridge: Cambridge Univ. Press, 1932.

5. Malin V. V. Theory of strip grating of finite period // Radio Eng. Electron. Phys. - 1963. - 8. - P. $185-193$.

6. Scarpetta E., Sumbatyan M. A. Explicit analytical results for one-mode normal reflection and transmission by a periodic array of screens // J. Math. Anal. and Appl. - 1995. - 195. - P. 736-749.

7. Scarpetta E., Sumbatyan M. A. Explicit analytical results for one-mode oblique penetration into a periodic array of screens // IMA J. Appl. Math. - 1996. - 56. - P. 109-120. 
8. Scarpetta E., Sumbatyan M. A. Wave propagation through a periodic array of inclined cracks // Eur. J. Mech. A/Solids. - 2000. - 19. - P. 949-959.

9. Shenderov Ye. L. Propagation of sound through a screen of arbitrary wave thickness with gaps // Sov. Phys. Acoust. - 1970. - 16, № 1. - P. $115-131$.

10. Solokhin N. V., Sumbatyan M. A. Artificial layer // Res. Nondestr. Eval. - 1994. - 6. - P. $19-34$.

11. Scarpetta E., Sumbatyan M. A. Wave penetration through elastic solids with a periodic array of rectangular flaws // Meccanica. - 2001. - 36. - P. 191-199.

12. Twersky V. On the scattering of waves by an infinite grating // IEEE Trans. Antennas and Propag. - 1956. 4. - P. $330-345$.

13. Miles J. W. On Rayleigh scattering by a grating // Wave Motion. - 1982. - 4. - P. $285-292$.

14. Van den Berg P. M., Voorman O. J. Diffraction by a grating of cylinders with an arbitrary cross-section // Appl. Sci. Res. - 1972. - 26. - P. 175-182.

15. Achenbach J. D. Wave propagation in elastic solids. - Amsterdam: North-Holland, 1973.

Received 15.01.2003 